H. Saito

Nagoya Math. J.

Vol. 101 (1986), 1-26

\title{
A NOTE ON CUBIC EQUIVALENCES
}

\author{
HIROSHI SAITO
}

\section{Dedicated to the memory of Professor Takehiko Miyata}

The present note is intended to be a supplement to [9], in which the following is proven: Let $V$ be a smooth projective variety over the field of complex numbers $C, T$ a smooth quasi-projective variety, $Z$ a cycle in $T \times V$ of codimension $p$. If $Z(t)$ is $\ell$-cube equivalent to zero for general $t \in T$, then, setting $r=\operatorname{dim} V-p$,

$$
\left\{{ }^{t} Z\right\}: H^{r}\left(V, \Omega_{V}^{r+\ell^{\prime}}\right) \longrightarrow H^{0}\left(T, \Omega_{T}^{\ell^{\prime}}\right)
$$

vanishes for $\ell^{\prime}<\ell$, where $\left\{{ }^{t} Z\right\}$ is the correspondence defined by $Z$.

If $r=0, H^{0}\left(V, \Omega_{V}^{\ell^{\prime}}\right)$ was classically called the space of integrals of the first kind on $V$, and we can ask: Is an analogue true for the integrals of the second kind? The aim of this note is to give an affirmative answer to this question (cf. (3.2)) if we take, as the definition of integrals of the second kind, that defined by Atiyah-Hodge [1]; $H^{r}\left(V, \Omega_{V}^{r+\ell^{\prime}}\right)$ is then replaced by the associated graded module for the coniveau filtration of $H^{2 r+\ell^{\prime}}(V)$.

A similar result holds also for etale cohomology and for varieties even over a positive characteristic ground field, but the definition of integrals of the second kind is, a priori at least, different from that by Grothendieck [5] (cf. (2.6.1)).

Anyway, for abelian surfaces which are not supersingular, the square equivalence and the cubic equivalence differ if the ground field is uncountable (cf. (3.6)). This should be compared with the following: if the ground field is the algebraic closure of a finite field, the square equivalence is the same as the rational equivalence for any variety, hence the square equivalence and the cubic equivalence are identical.

Again over the field of complex numbers, note that the space of integrals of the second kind carries a polarized Hodge structure. Then the corollary (3.5) should give some insight into the metaconjecture of Bloch [2], p. 1.14 .

Received July 21, 1983. 
In Section 1, we recall about the coniveau filtrations, and prove its functoriality (1.5). Section 2 is devoted to a proposition (2.5) on a family of products of curves, which is the key to the proof of the theorem (3.2). In Section 3, the results mentioned above are proven.

\section{§1. Review on coniveau filtrations}

(1.1) Let $X$ be a smooth quasi-projective variety over an algebraically closed field $k$. We denote by $X_{a}$ one of the following sites:

case a). char $k=0-X_{a}$ is the Zariski site of $X$;

case b). $k=C$, the field of complex numbers-. $X_{a}$ is the classical site of $X$;

case c). $k$ is arbitrary-. $X_{a}$ is the etale site of $X$.

In any case, we have the canonical morphism of sites

$$
\alpha_{X}=\alpha: X_{a} \longrightarrow X_{\mathrm{Zar}} .
$$

By $A$ and $\mu=\mu_{X}=\mu_{X}(a)$, we denote the following ring and the object of the derived category $D^{+}\left(X_{a}\right)$ of sheaves on $X_{a}$ :

in case $a), A=k$, and $\mu=\Omega_{X / k}^{*}$, the de Rham complex;

in case $b), A=Z$, and $\mu=Z$, the constant sheaf of $X_{a}$;

in case $c$ ), let $\nu$ be a prime number $\neq$ char $k, N>0$, and $a$ integers. We denote by $\mu_{\nu N}$ the etale sheaf of $\nu^{N}$-th roots of unity on $X_{a}$. Then $A=$ $Z / \nu^{N}$, and $\mu=\left(\mu_{\nu N}\right)^{\otimes a}$ if $a \geqslant 0$, and $\mu=\operatorname{Hom}_{A}\left(\left(\mu_{\nu N}\right)^{\otimes(-a)}, A\right)$ if $a<0$. (We often denote by the same letter $A$ the constant sheaf on $X_{a}$ defined by $A$ ).

For an integer $p \geqslant 0$, let $Z^{p}=Z^{p}(X)$ be the family of supports consisting of (Zariski) closed subsets of $X$ of codimension $\geqslant p$, and $\Gamma_{Z^{p}}\left(X_{a}\right.$, ?) the functor "sections with supports in $Z^{p}$ ". We have then, in the derived category of abelian groups, a sequence of triangles:

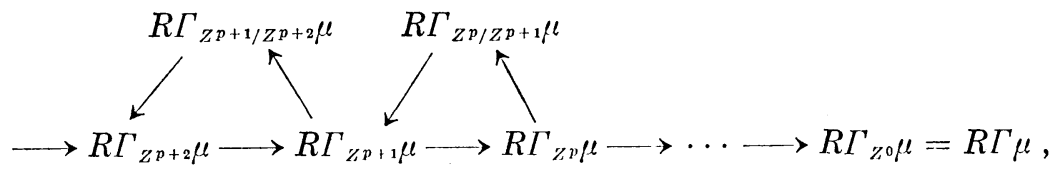

inducing the spectral sequence

$$
{ }_{N} E_{1}^{p, n-p}=R^{n} \Gamma_{Z^{p / Z^{p+1}}} \mu \Longrightarrow R^{n} \Gamma \mu .
$$


Let $\underline{Z}^{p}$ be the Zariski sheaf of supports obtained by localizing $Z^{p}$. Then, in the derived category $D^{+}\left(X_{\mathrm{zar}}\right)$ of Zariski sheaves on $X$, there exists a sequence of triangles

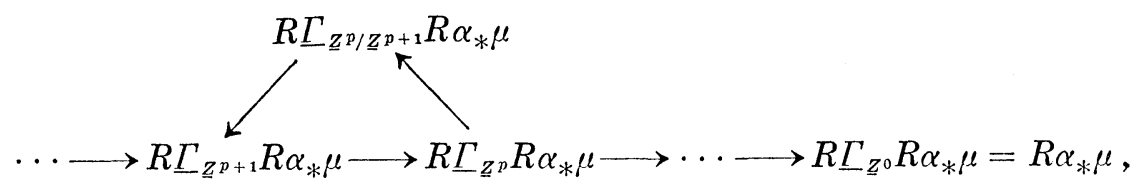

which induces the spectral sequence

$$
E_{1}^{p, n-p}=R^{n} \underline{\Gamma}_{\underline{Z}^{p} / \underline{Z}^{p+1}}\left(R \alpha_{*} \mu\right) \Longrightarrow R^{n} \alpha_{*} \mu .
$$

The application of $R \Gamma\left(X_{\mathrm{zar}}, ?\right)$ to (1.1.3) gives the sequence of triangles (1.1.1).

For a closed subset $Y$ of $X$, we shall denote $R^{n} \Gamma_{Y}(X, \mu)$ by $H_{Y}^{n}(X)$. Then

$$
R^{n} \Gamma_{Z p} \mu=H_{Z p}^{n}(X, \mu)=\lim _{\bar{Y} \in Z^{p}} H_{Y}^{n}(X),
$$

and

$$
R^{n} \Gamma_{Z^{p / Z p+1}} \mu=H_{Z^{p / Z^{p+1}}}^{n}(X, \mu)=\lim _{\substack{Y^{\prime} \in Z^{p} \\ Y^{\prime} \in Z^{p+1}}} H_{Y \backslash Y^{\prime}}^{n}\left(X \backslash Y^{\prime}\right) .
$$

The Zariski sheaves $R^{n} \underline{\Gamma}_{\underline{Z}}\left(R \alpha_{*} \mu\right)$ and $R^{n} \underline{\Gamma}_{\underline{Z}^{p / Z^{p+1}}}\left(R \alpha_{*} \mu\right)$ are obtained by sheafifying $H_{Z^{p}}^{n}(X, \mu)$ and $H_{Z^{p / Z^{p+1}}}^{n}(X, \mu)$ respectively with respect to the Zariski topology of $X$.

We shall denote by $N^{p} H^{n}(X, \mu)$ the filtration on the abutment $H^{n}(X, \mu)$ of the spectral sequence (1.1.2) which we call the coniveau filtration, and by $\operatorname{gr}^{p} H^{n}(X, \mu)$ its associated graded module:

$$
\operatorname{gr}^{p} H^{n}(X, \mu)=N^{p} H^{n}(X, \mu) / N^{p+1} H^{n}(X, \mu) .
$$

Explicitly,

$$
\begin{aligned}
N^{p} H^{n}(X, \mu) & =\operatorname{Im}\left(H_{Z p}^{n}(X, \mu) \longrightarrow H^{n}(X, \mu)\right) \\
& =\sum_{Y \in Z^{p}} \operatorname{Im}\left(H_{Y}^{n}(X, \mu) \longrightarrow H^{n}(X, \mu)\right) \\
& =\sum_{Y \in Z^{p}} \operatorname{Ker}\left(H^{n}(X, \mu) \longrightarrow H^{n}(X \backslash Y, \mu)\right) .
\end{aligned}
$$

(Since $Z^{p}$ is filtering for inclusion, we may replace $\sum$ by $U$.)

Let $Z^{(p)}$ denote the points of $X$ of codimension $p$. Then we have

$$
R^{n} \underline{\Gamma}_{\underline{Z}^{p} / \underline{Z}^{p+1}}\left(R \alpha_{*} \mu\right)=\prod_{x \in Z^{(p)}} i_{x} H_{x}^{n}\left(R \alpha_{*} \mu\right)
$$


where $i_{x} H_{x}^{n}\left(R \alpha_{*} \mu\right)$ is the direct image sheaf of $H_{x}^{n}\left(R \alpha_{*} \mu\right)$ regarded as a constant sheaf on the space $\{x\}$ by the inclusion from $\{x\}$ into $X$, see [6]. Hence that sheaf is flasque. For more explicit formula of $H_{x}^{n}\left(R \alpha_{*} \mu\right)$, see [3].

Theorem (1.2) (Bloch-Ogus, [3], 4.2). The spectral sequence (1.1.4) degenerates from $E_{2}$-term on, and $E_{2}^{p, q}=0$ for $p \neq 0$.

Theorem (1.3) (Bloch-Ogus-Deligne). The spectral sequence (1.1.2) is isomorphic to the Leray spectral sequence

$$
E_{2}^{p, q}=H^{p}\left(X_{\mathrm{zar}}, R^{q} \alpha_{*} \mu\right) \Longrightarrow H^{n}\left(X_{a}, \mu\right)
$$

from $E_{2}$-term on. In particular, the filtrations of $H^{n}\left(X_{a}, \mu\right)$ by these spectral sequences coincide.

In case a), the theorem (1.3) was proven in [3]. In cases b) and c), according to the footnote (loc. cit.), it was shown by Deligne. For completeness, we give a proof here.

Lemma (1.4). Let $\mathscr{A}$ and $\mathscr{B}$ be abelian categories, $\mathscr{A}$ having enough injectives, $T: \mathscr{A} \rightarrow \mathscr{B}$ a left exact additive functor, $K=F^{0}, F^{1}, \cdots, F^{m}$ objects of $D^{+}(\mathscr{A}), F^{p+1} \rightarrow F^{p}$ morphisms. We have a sequence of triangles:

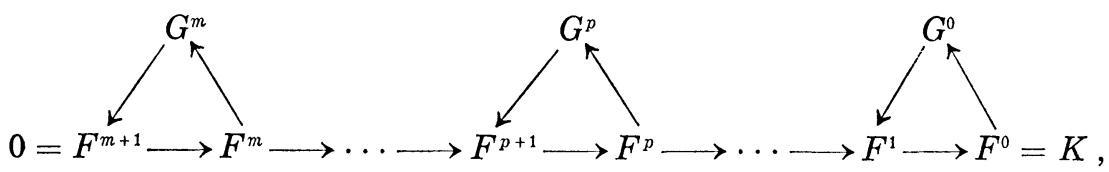

inducting a spectral sequence

$$
E_{1}^{p, n-p}=H^{n}\left(G^{p}\right) \Longrightarrow H^{n}(K) .
$$

Assume that

(i ) $E_{1}^{p, q}$ are $T$-acyclic,

(ii) $E_{2}^{p, q}=0$ for $p \neq 0$.

Applying the functor $R T$ to the sequence of triangles above, we get

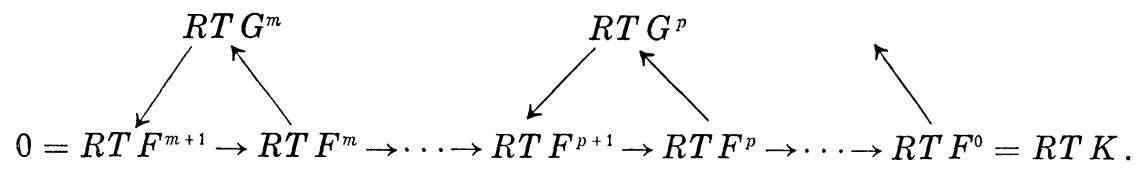

Then the spectral sequence $E_{1}^{p, n-p}=R^{n} T G^{p} \Rightarrow R^{n} T K$ obtained by this sequence of triangles is isomorphic to the second spectral sequence 


$$
E_{2}^{p, q}=R^{p} T H_{q}(K) \Longrightarrow R^{n} T K,
$$

from $E_{2}$-term on.

The lemma (1.4) implies the theorem (1.3): We apply the lemma for $\mathscr{A}=$ the category of sheaves of abelian groups on $X_{\mathrm{zar}}, \mathscr{B}=$ the category of abelian groups, $T=\Gamma\left(X_{\mathrm{zar}}\right.$, ?), $F^{p}=R \underline{\Gamma}_{\underline{Z}^{p}}\left(R \alpha_{*} \mu\right)$, and $G^{p}=R \underline{\underline{Z}}_{\underline{Z}^{p / Z} \underline{Z}^{p+1}}\left(R \alpha_{*} \mu\right)$. Then (i) holds as noticed above, and (ii) is satisfied by (1.2). And the second spectral sequence for $R \Gamma\left(X_{\mathrm{zar}}, R \alpha_{*} \mu\right)$ is nothing but the Leray spectral sequence for $\alpha$.

Before giving the proof of (1.4), we shall prove the elementary

Lemma (1.4.1). Let $\mathscr{A}$ be an abelian category, $f: A^{\cdot} \rightarrow I^{\cdot}$ be a morphism of complexes bounded below in $\mathscr{A}$ such that

(i) $f_{*}: H^{n}\left(A^{*}\right) \rightarrow H^{n}\left(I^{*}\right)$ are zero for all $n$;

(ii) $H^{n}\left(I^{\bullet}\right)$ and $\operatorname{Im}\left(d^{n-1}: I^{n-1} \rightarrow I^{n}\right)$ are injective objects in $\mathscr{A}$ for all $n$. Then, $f$ is homotopic to zero.

We construct a homotopy $k^{n}: A^{n+1} \rightarrow I^{n}$ by induction on $n$. For $n \ll 0$, it is necessarily zero since so are $A^{n}$ and $I^{n}$. We have exact sequences

$$
\begin{aligned}
& 0 \longrightarrow B_{I}^{n} \longrightarrow Z_{I}^{n} \longrightarrow H_{I}^{n} \longrightarrow 0 . \\
& 0 \longrightarrow Z_{I}^{n} \longrightarrow I^{n} \longrightarrow B_{I}^{n+1} \longrightarrow 0,
\end{aligned}
$$

where $B_{I}^{n}=\operatorname{Im}\left(d: I^{n-1} \rightarrow I^{n}\right), \quad Z_{I}^{n}=\operatorname{Ker}\left(d: I^{n} \rightarrow I^{n+1}\right)$, and $H_{I}^{n}=H^{n}\left(I^{*}\right)$. By hypothesis, $B_{I}^{n}$ and $H_{I}^{n}$ are injective, so that we have

$$
\begin{aligned}
& Z_{I}^{n} \cong B_{I}^{n} \oplus H_{I}^{n}, \\
& I^{n} \cong Z_{I}^{n} \oplus B_{I}^{n+1} \cong B_{I}^{n} \oplus H_{I}^{n} \oplus B_{I}^{n+1} .
\end{aligned}
$$

Denoting the coboundary and cocycle of $A^{\cdot}$ by $B^{n}$ and $Z^{n}$, we get a commutative diagram with exact rows:

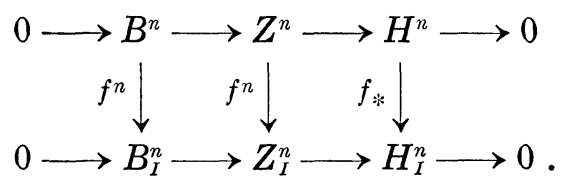

Since $f_{*}=0, f^{n}: Z^{n} \rightarrow Z_{I}^{n}$ factors through $B_{I}^{n}$ :

$$
f^{n}: Z^{n} \longrightarrow B_{I}^{n} \longrightarrow Z_{I}^{n} .
$$

Notice that giving $f^{n}: A^{n} \rightarrow I^{n} \cong B_{I}^{n} \oplus H_{I}^{n} \oplus B_{I}^{n+1}$ is equivalent to giving $\mathrm{pr}_{1} \circ f^{n}, p r_{2} \circ f^{n}, \mathrm{pr}_{3} \circ f^{n}$, where $\mathrm{pr}_{i}$ are projections. Since the diagram 


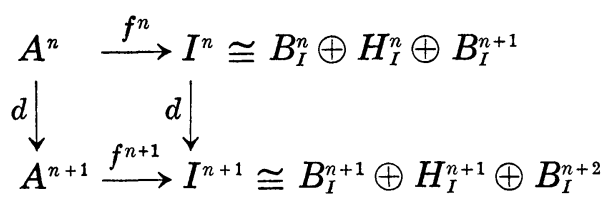

is commutative, and since $d: I^{n} \rightarrow I^{n+1}$ is given by $I^{n} \stackrel{\mathrm{pr}_{3}}{\longrightarrow} B_{I}^{n+1} \smile I^{n+1}$,

$$
\mathrm{pr}_{3} \circ f^{n}=\mathrm{pr}_{1} \circ f^{n+1} \circ d \text {. }
$$

Now suppose that we have constructed $k^{i+1}: A^{i+1} \rightarrow H_{I}^{i} \oplus B_{I}^{i+1} \subset I^{i}$ for $i<n$ such that

$$
\begin{array}{ll}
d \circ k^{i}+k^{i+1} \circ d=f^{i} & (i<n), \\
\mathrm{pr}_{1} \circ f^{i}=\mathrm{pr}_{1} \circ d \circ k^{i} & (i \leqslant n) .
\end{array}
$$

Define $\beta: A^{n+1} \rightarrow B_{I}^{n+2}$ by

$$
\beta: A^{n+1} \stackrel{f^{n+1}}{\longrightarrow} I^{n+1} \stackrel{\mathrm{pr}_{1}}{\longrightarrow} B_{I}^{n+1} .
$$

The restriction to $Z^{n}$ of

$$
A^{n} \stackrel{f^{n}}{\longrightarrow} I^{n} \stackrel{\mathrm{pr}_{2}}{\longrightarrow} H_{I}^{n}
$$

is zero by hypothesis. Taking the quotient, we get $B^{n+1} \rightarrow H_{I}^{n}$. Since $H_{I}^{n}$ is injective, it can be extended to $\alpha: A^{n+1} \rightarrow H_{I}^{n}$. We set

$$
k^{n+1}=(\alpha, \beta): A^{n+1} \longrightarrow H_{I}^{n} \oplus B_{I}^{n+1} \subset I^{n} .
$$

Then we have $f^{n}=k^{n+1} \circ d+d \circ k^{n}, \mathrm{pr}_{1} \circ f^{n+1}=\mathrm{pr}_{1} \circ d \circ k^{n+1}$, which completes the proof of (1.4.1).

Now we prove the lemma (1.4). Let $K^{\bullet}$ be a complex "representing" $K$ in $D^{+}(\mathscr{A})$, and $K^{*} \rightarrow I^{*}$ be a Cartan-Eilenberg resolution of $K^{*}, \tau_{\mathrm{I} I \geqslant p} I^{*}$ denote the filtration of the complex $I^{*}$ defined by

$$
\tau_{\mathrm{II} \geqslant p} I^{a, b}= \begin{cases}0 & (b<p) \\ I^{a, b} & (b \geqslant p) .\end{cases}
$$

We shall construct morphisms $\delta^{p}: F^{p} \rightarrow \tau_{\mathrm{II} \geqslant p} I^{\cdot \bullet}$ in $D^{+}(\mathscr{A})$ such that

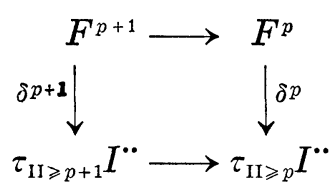

commutes, where the horizontal arrow below is the "inclusion". For $p=0$, 
$\delta^{p}$ is $K^{\cdot} \rightarrow I^{\bullet}$. Suppose $\delta^{p}: F^{p} \rightarrow \tau_{\mathrm{II} \geqslant p} I^{\bullet}$ is constructed, and set $\alpha: F^{p+1} \rightarrow$ $F^{p} \stackrel{\delta^{p}}{\longrightarrow} \tau_{\mathrm{II} \geqslant p} I^{*}$. The condition (ii) is equivalent to the condition that $H^{n}\left(F^{p+1}\right) \rightarrow H^{n}\left(F^{p}\right)$ are zero for all $n$. In fact, it results from the condition (ii) that $E_{\infty}^{p, q}=0$ for $p \neq 0$, hence $F^{1} H^{n}(K)=0$, i.e., the map $H^{n}\left(F^{1}\right) \rightarrow$ $H^{n}\left(F^{0}\right)$ is zero. The spectral sequence induced by the sequence $\cdots \rightarrow F^{i+1}$ $\rightarrow F^{i} \rightarrow \cdots \rightarrow F^{p}$ is also degenerate at $E_{2}$-term by virtue of (ii), and we conclude as above that $H^{n}\left(F^{p+1}\right) \rightarrow H^{n}\left(F^{p}\right)$ is zero. The converse is easy to see. Hence,

$$
\alpha_{*}: H^{n}\left(F^{p+1}\right) \longrightarrow H^{n}\left(\tau_{\mathrm{II} \geqslant p} I^{\bullet}\right)
$$

is zero for every $n$. We have the triangle

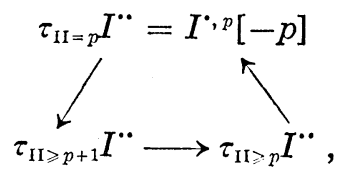

so that the sequence

$$
\operatorname{Hom}\left(F^{p+1}, \tau_{\mathrm{II} \geqslant p+1} I^{\cdot *}\right) \longrightarrow \operatorname{Hom}\left(F^{p+1}, \tau_{\mathrm{II} \geqslant p} I^{\cdot *}\right) \stackrel{r}{\longrightarrow} \operatorname{Hom}\left(F^{p+1}, \tau_{\mathrm{II} \geqslant p} I^{\cdot *}\right)
$$

is exact. Now $H^{n}\left(\tau_{\mathrm{II}=p} I^{*}\right)$ and $\operatorname{Im}\left(d:\left(\tau_{\mathrm{II}=p} I^{\cdot \bullet}\right)^{n-1} \longrightarrow\left(\tau_{\mathrm{II}=p} I^{\cdot \bullet}\right)^{n}\right)$ are injective objects for all $n$, and from what noted above,

$$
(\gamma(\alpha))_{*}: H^{n}\left(F^{p+1}\right) \longrightarrow H^{n}\left(\tau_{\mathrm{II}=p} I^{\bullet *}\right)
$$

are zero for all $n$. By virtue of (1.4.1), $\gamma(\alpha): F^{p+1} \rightarrow \tau_{\mathrm{II}=p} I^{*}$ is homotopic to zero, or $\gamma(\alpha)=0$ in $D^{+}(\mathscr{A})$. Thus, by the exactness of the sequence above, there exists a morphism $\delta^{p+1}: F^{p+1} \rightarrow \tau_{\mathrm{II} \geqslant p+1} I^{\cdot \cdot}$ making the diagram (1.4.2) commutative. Therefore, we have a commutative diagram

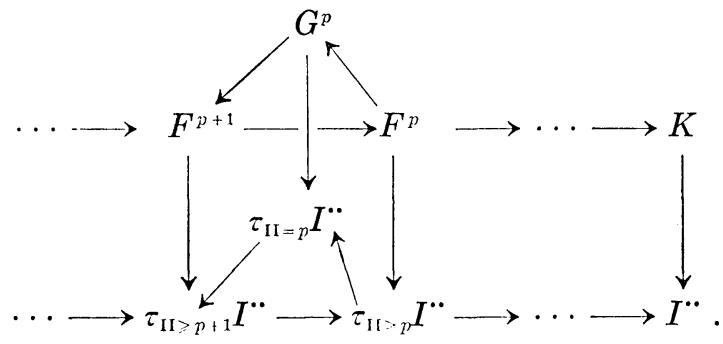

Applying $R T$, we get: 


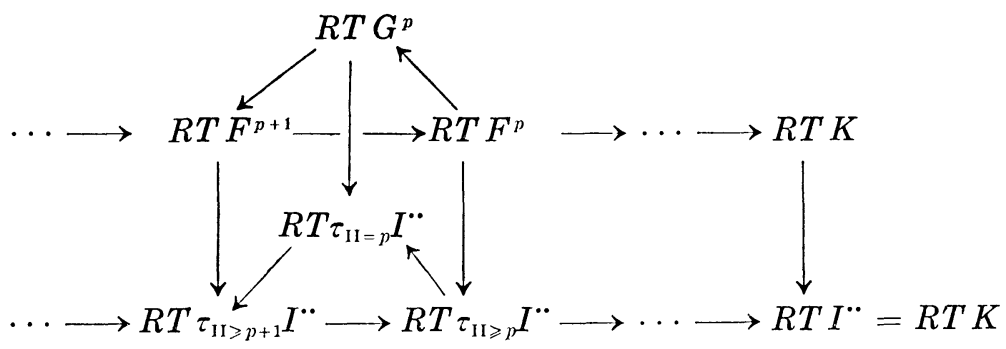

Denote by ${ }_{{ }_{T}} E_{r}^{p, q}$ and ${ }_{{ }_{\mathrm{II}} I} E_{r}^{p, q}$ the spectral sequences obtained by the sequences of triangles above and below respectively. Hence we have a morphism of spectral sequences

$$
{ }_{T F} E_{r}^{p, q} \longrightarrow{ }_{{ }_{\mathrm{II} I}} E_{r}^{p, q} .
$$

We shall show that it is an isomorphism on $E_{2}$-term, which completes the proof, since ${ }_{T_{\mathrm{II}} I} E_{r}^{p, q}$ is, by definition, the second spectral sequence for $R T K$.

Consider spectral sequences

$$
\begin{aligned}
& R^{a} T H^{b}\left(G^{p}\right) \Longrightarrow R^{a+b} T G^{p} \\
& R^{a} T H^{b}\left(\tau_{\mathrm{II}=p} I^{\bullet \cdot}\right) \Longrightarrow R^{a+b} T\left(\tau_{\mathrm{II}=p} I^{\bullet \cdot}\right),
\end{aligned}
$$

and a morphism of spectral sequences from the above to the below induced by $G^{p} \rightarrow \tau_{\mathrm{II}=p} I^{*}$. Since $H^{b}\left(G^{p}\right)$ are $T$-acyclic by hypothesis, and $H^{b}\left(\tau_{\mathrm{II}=p} I^{* \bullet}\right)$ are injective, hence $T$-acyclic, we get

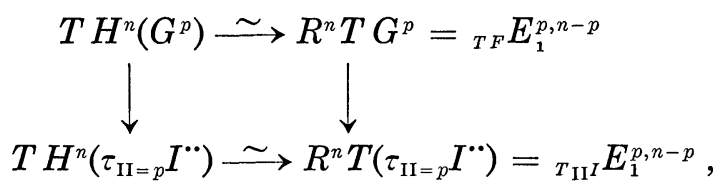

so that it suffices to show that the morphism of complexes

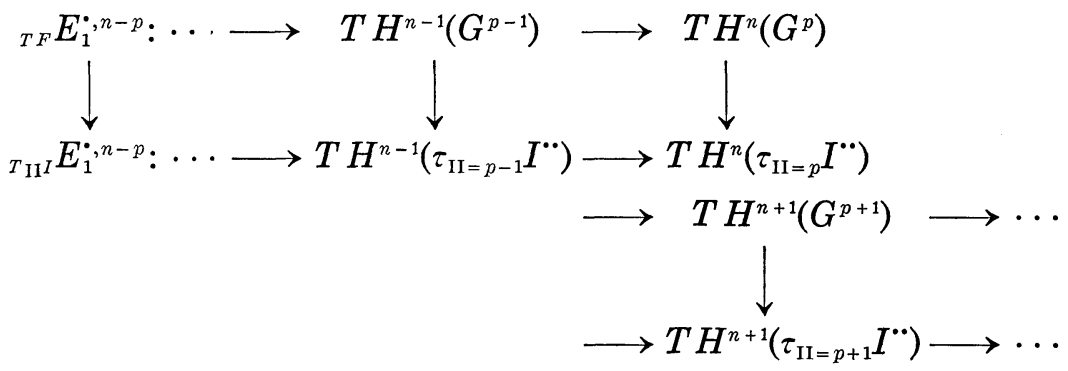


is a quasi-isomorphism. This morphism is, however, obtained by applying $T$ to the morphism of complexes:

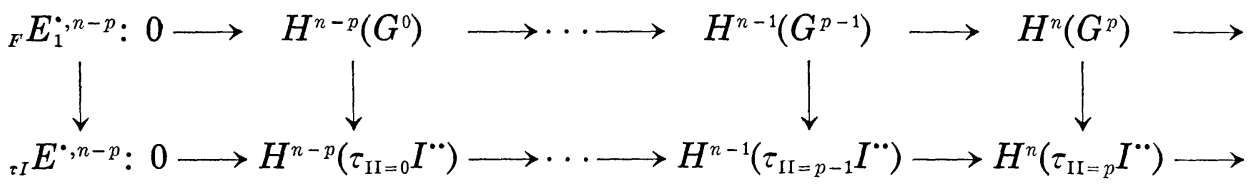

By hypotheses, ${ }_{F} E_{1}^{\cdot, n-p}$ is a $T$-acyclic resolution of $H^{n-p}(K)$ and ${ }_{\tau I} E_{1}^{\cdot, n-p}$ is an injective resolution of $H^{n-p}\left(I^{*}\right) \simeq H^{n-p}(K)$, hence their cohomologies are isomorphic:

$$
{ }_{T F} E_{2}^{p, n-p} \simeq R^{p} T H^{n-p}(K) \simeq{ }_{{ }_{\mathrm{III}}} E_{2}^{p, n-p} .
$$

Corollary (1.5). (i) For a morphism $f: X \rightarrow Y$, we have the pullback

$$
f^{*}: H^{n}\left(Y, \mu_{Y}\right) \longrightarrow H^{n}\left(X, \mu_{X}\right) \text {. }
$$

Then, $f^{*}\left(N^{*} H^{n}\left(Y, \mu_{Y}\right)\right) \subset N^{p} H^{n}\left(X, \mu_{X}\right)$. Therefore, by taking the quotient, we get

$$
f^{*}: \operatorname{gr}^{p} H^{n}\left(Y, \mu_{Y}\right) \longrightarrow \operatorname{gr}^{p} H^{n}\left(X, \mu_{X}\right) \text {. }
$$

(ii) For a proper morphism $f: X \rightarrow Y$, we have a Gysin map

$$
f_{*}: H^{n}\left(X, \mu_{X}(a)\right) \longrightarrow H^{n-2 d}\left(Y, \mu_{Y}(a-d)\right),
$$

where $d=\operatorname{dim} X-\operatorname{dim} Y$. Then,

$$
f_{*}\left(N^{p} H^{n}\left(X, \mu_{X}(a)\right)\right) \subset N^{p-d} H^{n-2 d}\left(Y, \mu_{Y}(a-d)\right),
$$

hence we get

$$
f_{*}: \operatorname{gr}^{p} H^{n}\left(X, \mu_{X}(a)\right) \longrightarrow \operatorname{gr}^{p-d} H^{n-2 d}\left(Y, \mu_{Y}(a-d)\right) .
$$

(iii) By the cup-product

$$
\cup: H^{n}\left(X, \mu_{X}(a)\right) \times H^{n^{\prime}}\left(X, \mu_{X}\left(a^{\prime}\right)\right) \longrightarrow H^{n+n^{\prime}}\left(X, \mu_{X}\left(a+a^{\prime}\right)\right),
$$

$N^{p} H^{n}\left(X, \mu_{X}(a)\right) \times N^{p} H\left(X,\left(a^{\prime}\right)\right)$ is mapped into $N^{p+p^{\prime}} H^{n+n^{\prime}}\left(X, \mu_{X}\left(a+a^{\prime}\right)\right)$. Hence, we obtain

$$
\cup: \operatorname{gr}^{p} H^{n}\left(X, \mu_{X}(a)\right) \times \operatorname{gr}^{p^{\prime}} H^{n^{\prime}}\left(X, \mu_{X}\left(a^{\prime}\right)\right) \longrightarrow \operatorname{gr}^{p+p^{\prime}} H^{n+n^{\prime}}\left(X, \mu_{X}\left(a+a^{\prime}\right)\right) .
$$

(ii) is trivial and well-known (cf. [3]). We shall prove (i) and (iii). Let $f: X \rightarrow Y$ be a morphism. We have a commutative diagram of sites 


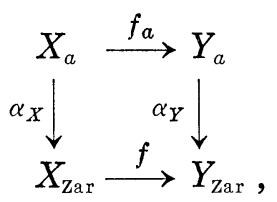

and we get a morphism $f^{*}$ of Leray spectral sequences from

$$
E_{2}^{p, n-p}=H^{p}\left(Y_{\mathrm{Zar}}, R^{n-p} \alpha_{Y} * \mu_{Y}\right) \Longrightarrow H^{n}\left(Y_{a}, \mu_{Y}\right)
$$

to

$$
E_{2}^{p, n-p}=H^{p}\left(X_{\mathrm{Zar}}, R^{n-p} \alpha_{X^{*}} \mu_{X}\right) \Longrightarrow H^{n}\left(X_{a}, \mu_{X}\right) .
$$

Since the coniveau filtration coincides with the filtration by Leray spectral sequence, we get (i). As for the cup-product, we have a pairing of spectral sequences (cf. [4], p. 336).

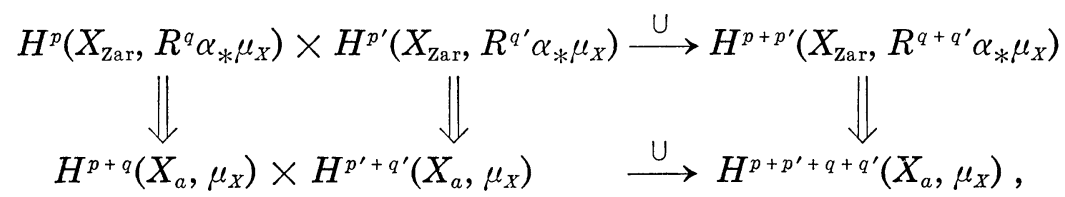

(We have omitted symbols of twisting for simplicity). Whence (iii).

Remarks (1.5.1). For an (algebraic) cycle $Z$ on $X$ of codimension $p$, we have the fundamental class

$$
\{Z\} \in H^{2 p}\left(X_{a}, \mu_{X}(p)\right) \text {. }
$$

The subgroup $N^{p} H^{2 p}\left(X_{a}, \mu_{X}(p)\right) \simeq \operatorname{gr}^{p} H^{2 p}\left(X_{a}, \mu_{X}(p)\right)$ is generated by the fundamental classes over $A$. The operation $Z \mapsto\{Z\}$ is compatible with pull-backs, direct images, and the products. An equality of maps between cohomologies induced by algebraic correspondences carries over to their associated graded modules by the corollary (1.5). For example, let $Z$ be a cycle on $X \times Y$, and suppose that we have an equality of the type

$$
0=\{Z\}: H^{n}(X) \longrightarrow H^{n+2 k}(Y) ;
$$

then it induces

$$
0=\{Z\}: \operatorname{gr}^{p} H^{n}(X) \longrightarrow \operatorname{gr}^{p+k} H^{n+2 k}(Y) .
$$

(1.5.2). As for (1.5), (ii), we have also a morphism of spectral sequences

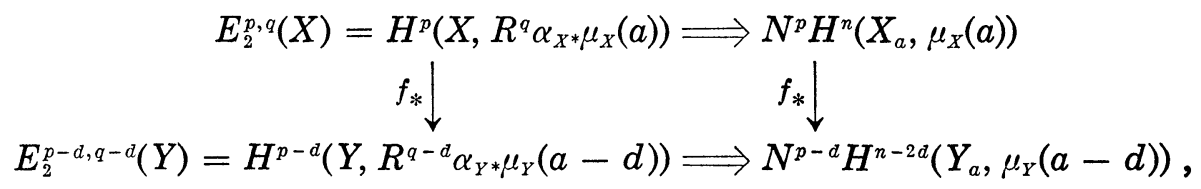


where $E_{r}^{p-d, q-d}(Y)$ is the shift of the spectral sequence $E^{p, q}(Y)$ by $(-d,-d)$. In the case a), for example, we have isomorphisms

$$
\begin{aligned}
E_{2}^{p, p}(X) \simeq & \text { (the algebraic cycles on } X \text { of codimension } p \text { modulo algebraic } \\
& \text { equivalence }) \otimes k, \\
E_{\infty}^{p, p}(X) \simeq & \text { (the algebraic cycles on } X \text { of codimension } p \text { modulo homo- } \\
& \text { logical equivalence }) \otimes k .
\end{aligned}
$$

Since $E_{2}^{p, q}=0$ for $p>q$, we have natural surjective maps $E_{2}^{p, p} \rightarrow E_{r}^{p, p}$, so that

$$
\begin{aligned}
E_{r}^{p, p}(X) \simeq & \text { (the algebraic cycles on } X \text { of codimension } p \text { modulo some } \\
& \text { equivalence relation }) \otimes k .
\end{aligned}
$$

In view of morphisms of spectral sequences above, this equivalence relation is compatible with pull-backs, direct images and the intersection products, hence an adequate equivalence relation. Thus we have a "filtration of adequate equivalence relations" between algebraic and homological equivalences, which stops at most at $(p-1)$-th step for cycles of codimension $p$.

Lemma (1.6). If $f: X \longrightarrow Y$ is an open immersion, then

$$
f^{*}: \operatorname{gr}^{0} H^{n}\left(Y, \mu_{Y}\right) \longrightarrow \operatorname{gr}^{0} H^{n}\left(X, \mu_{X}\right)
$$

is injective for every $n$.

Immediate from the last expression of (1.1.7).

Lemma (1.7). If $f: X \rightarrow Y$ is a dominant morphism, then the kernel of the map

$$
f^{*}: \operatorname{gr}^{0} H^{n}\left(Y, \mu_{Y}\right) \longrightarrow \operatorname{gr}^{0} H^{n}\left(X, \mu_{X}\right)
$$

is killed by an integer $\neq 0$ (independent of $n$, and of $\nu, a$, and $N$ in the case c)).

Let $X^{\prime}$ be a (locally closed) subvariety of $X$ such that

$$
f^{\prime}: X^{\prime} \longrightarrow X \stackrel{f}{\longrightarrow} Y
$$

is generically finite and dominant. If necessary, by shrinking $X^{\prime}$, we may assume that $X^{\prime}$ is smooth. We have

$$
\begin{aligned}
& \operatorname{Ker}\left(f^{*}: \operatorname{gr}^{0} H^{n}(Y, \mu) \longrightarrow \operatorname{gr}^{0} H^{n}(X, \mu)\right) \\
& \quad \subset \operatorname{Ker}\left(f^{*}: \operatorname{gr}^{0} H^{n}(Y, \mu) \longrightarrow \operatorname{gr}^{0} H^{n}\left(X^{\prime}, \mu\right)\right) ;
\end{aligned}
$$

hence we may suppose that $f$ is generically finite. Let $d$ be the degree of $f$. If $f$ is proper, the projection formula shows that 


$$
H^{n}(Y, \mu) \stackrel{f^{*}}{\longrightarrow} H^{n}(X, \mu) \stackrel{f_{*}}{\longrightarrow} H^{n}(Y, \mu)
$$

is the multipliction by $d$, hence by taking the associated graded module, we get the lemma. In general case, there exists a nonempty open subvariety $Y_{0}$ of $Y$ such that the restriction $f_{0}=f_{\left(Y_{0}\right)}: f^{-1}\left(Y_{0}\right)=X_{0} \rightarrow Y_{0}$ is proper. Since the diagram

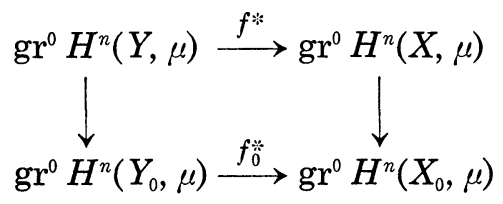

commutes and since the vertical maps are injective by (1.6), we have

$$
\operatorname{Ker} f^{*} \longrightarrow \operatorname{Ker} f_{0}^{*} \text {. }
$$

Then

$$
0=d \cdot \operatorname{Ker} f_{0}^{*} \longleftrightarrow d \cdot \operatorname{Ker} f^{*} . \quad \text { q.e.d. }
$$

\section{$\S 2$. A proposition on a family of products of curves}

(2.1) The aim of this section is to prove the proposition (2.5).

Since the Zariski topology is not so fine, we must treat the case a) and the cases b) and c) separately.

(2.2) First we consider the case a). Let $S$ be a smooth scheme over $k, h: X \rightarrow S$ a smooth morphism. On $\Omega_{X}^{*}=\Omega_{X / k}^{*}$, we have a filtration $F^{p} \Omega_{X}^{*}$ defined by

$$
F^{p} \Omega_{X}^{\cdot}=\operatorname{Im}\left(\Omega_{X}^{-p} \otimes_{O_{S}} \Omega_{S}^{p} \longrightarrow \Omega_{X}^{*}\right)
$$

and its associated graded object $\mathrm{Gr}^{p} \Omega_{X}^{*}$ is given by $\Omega_{X / S}^{\cdot-p} \otimes \Omega_{S}^{p}$, where $\Omega_{X}^{-p}$ and $\Omega_{X / S}^{*}$ denote the shifts by $p$ places to the right of $\Omega_{X}^{*}$ and $\Omega_{X / S}^{*}$ respectively. We obtain a spectral sequence

$$
{ }_{h} E: E_{1}^{p, n-p}=R^{n} h_{*}\left(\mathrm{Gr}^{p} \Omega_{X}^{*}\right)=R^{n-p} h_{*}\left(\Omega_{X / S}^{*}\right) \otimes \Omega_{S}^{p} \Longrightarrow R^{n} h_{*}\left(\Omega_{X}^{*}\right) .
$$

The filtration on the abutment $R^{n} h_{*}\left(\Omega_{X}^{*}\right)$ will be denoted by $F^{p} R^{n} h_{*}\left(\Omega_{X}^{*}\right)$ $={ }_{n} F^{p} R^{n} h_{*}\left(\Omega_{X}^{*}\right)$. Let $g: Y \rightarrow S$ be a smooth morphism, $s: Y \rightarrow X$ an $S$ morphism:

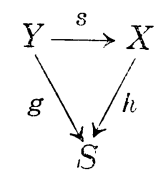


Then we have also a spectral sequence for $g$

$$
{ }_{g} E: E_{1}^{p, n-p}=R^{n-p} g_{*}\left(\Omega_{Y / S}^{\cdot}\right) \otimes \Omega_{S}^{*} \Longrightarrow R^{n} g_{*}\left(\Omega_{Y}^{*}\right)
$$

and the morphism $s^{*}$ of spectral sequences ${ }_{h} E \rightarrow{ }_{g} E$. Its $E_{1}$-term is given by $s^{*}: R^{n-p} h_{*}\left(\Omega_{X / S}^{*}\right) \rightarrow R^{n-p} g_{*}\left(\Omega_{Y / S}^{*}\right)$ tensored with $\Omega_{S}^{p}$.

(2.3) Now we consider the cases b) and c). Let $h: X \rightarrow S$ be a smooth morphism. Then we get a commutative diagram of sites:

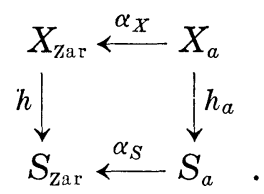

We have the Leray spectral sequence for $\alpha_{S} \circ h_{a}$ :

$$
{ }_{h} E: E_{2}^{p, q}=R^{p} \alpha_{S^{*}} R^{q} h_{a^{*}}\left(\mu_{X}\right) \Longrightarrow R^{n}\left(\alpha_{S} \circ h_{a}\right)_{*} \mu_{X} .
$$

The filtration on the abutment $R^{n}\left(\alpha_{S} \circ h_{a}\right)_{*} \mu_{X}$ will be denoted by $F^{p} R^{n}\left(\alpha_{S} \circ h_{a}\right)_{*} \mu_{X}={ }_{n} F^{p} R^{n}\left(\alpha_{S} \circ h_{a}\right)_{*} \mu_{X}$. Let $g: Y \rightarrow S$ be a morphism, $s: Y$ $\rightarrow X$ an $S$-morphism, hence we get a commutative diagram

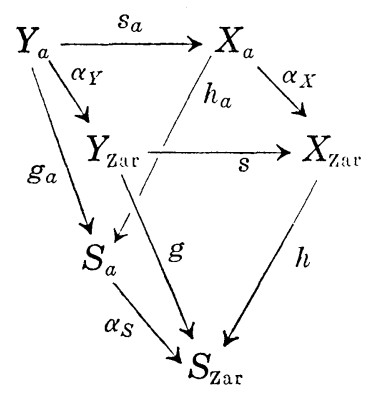

Then we have also the Leray spectral sequence

$$
{ }_{g} E: E_{2}^{p, q}=R^{p} \alpha_{S^{*}} R^{q} g_{a *} \mu_{Y} \Longrightarrow R^{n}\left(\alpha_{S} \circ g_{a}\right)_{*} \mu_{Y}
$$

and a morphism of spectral sequence $s^{*}:{ }_{h} E \rightarrow{ }_{g} E$. Its $E_{2}$-term is obtained by applying $R^{p} \alpha_{S^{*}}$ to $s^{*}: R^{q} h_{a *} \mu_{X} \rightarrow R^{q} g_{a^{*}} \mu_{Y}$.

Note that also in the case a), $R^{n}\left(\alpha_{S} \circ h_{a}\right)_{*} \mu_{X}=R^{n} h_{*}\left(\Omega_{X}^{*}\right)$, and we can consider the Leray spectral sequence (2.3.1), for which $E_{2}^{p, q}=0$ for $p \neq 0$. So it is a little bit confusing to employ the same notation ${ }_{n} E$ for the spectral sequences (2.2.1) and (2.3.1), but will be convenient as seen in the sequel. By the notation ${ }_{n} E$ (etc), we understand the spectral sequence 
(2.2.1) in the case a), and (2.3.1) in the cases b) and c), both abuting to $R^{n}\left(\alpha_{S} \circ h_{a}\right)_{*} \mu_{X}$.

By a family of curves $f: X \rightarrow S$, we mean that $f$ is smooth proper of relative dimension 1 with $f_{*}\left(O_{X}\right)=O_{S}$.

Lemma (2.4). Let $g: Y \rightarrow S$ be a smooth proper morphism of smooth schemes over $k, f^{\prime}: \mathscr{C} \rightarrow S$ a family of curves, $X=Y \underset{S}{\times} \mathscr{C}$ :

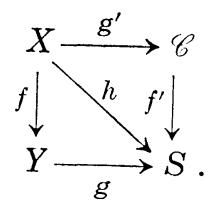

We set $h=g \circ f$ and suppose that two sections $s_{1}$ and $s_{2}$ of $\mathscr{C} / S$ are given, and let $\bar{s}_{1}, \bar{s}_{2}$ be the base changes of $s_{1}$ and $s_{2}$ by $g: Y \rightarrow S$. We have maps

$$
\bar{s}_{i}^{*}: R^{n}\left(\alpha_{S} \circ h_{a}\right)_{*} \mu_{X} \longrightarrow R^{n}\left(\alpha_{S} \circ g_{a}\right)_{*} \mu_{Y} \text {. }
$$

Then,

$$
\left(\bar{s}_{1}^{*}-\bar{s}_{2}^{*}\right)_{h} F^{p} R^{n}\left(\alpha_{S} \circ h_{a}\right)_{*} \mu_{X} \subset{ }_{g} F^{p+1} R^{n}\left(\alpha_{S} \circ g_{a}\right) \mu_{Y} .
$$

In fact we have a commutative diagram

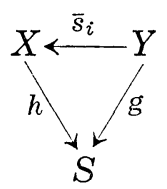

hence morphisms of spectral sequences $\bar{s}_{i}^{*}:{ }_{h} E \rightarrow{ }_{g} E$. It suffices to show $\bar{s}_{1}^{*}=\bar{s}_{2}^{*}$ on $E_{\infty}^{p, q}$, a fortiori on $E_{r}^{p, q}$. In the case a), it is therefore enough to verify

$$
\bar{s}_{1}^{*}=\bar{s}_{2}^{*}: R^{q} h_{*}\left(\Omega_{X / S}^{*}\right) \longrightarrow R^{q} g_{*}\left(\Omega_{Y / S}^{*}\right) .
$$

Since $X=Y \underset{S}{\times} \mathscr{C}$, by Künneth formula,

$$
R h_{*}\left(\Omega_{X / S}^{\cdot}\right)=R g_{*}\left(\Omega_{Y / S}^{\cdot}\right) \bigotimes_{O_{S}}^{L} R f_{*}^{\prime}\left(\Omega_{\mathscr{G} / S}^{\cdot}\right) .
$$

By [7], $R^{n} g_{*}\left(\Omega_{Y / S}^{*}\right)$ and $R^{n} f_{*}^{\prime}\left(\Omega_{\mathscr{C} / S}^{*}\right)$ are locally free of finite rank, hence,

$$
R^{n} h_{*}\left(\Omega_{X / S}^{\cdot}\right)=\underset{a+b=n}{\oplus} R^{a} g_{*}\left(\Omega_{Y / S}^{\cdot}\right) \underset{O_{S}}{\otimes} R^{b} f_{*}^{\prime}\left(\Omega_{\mathscr{C} / S}^{\cdot}\right)
$$

On

$$
R^{n} g_{*}\left(\Omega_{Y / S}^{\cdot}\right) \underset{O_{S}}{\otimes^{0}} R_{*}^{\prime}\left(\Omega_{\mathscr{G} / S}^{\cdot}\right) \simeq R^{n} g_{*}\left(\Omega_{Y / S}^{\cdot}\right) \underset{O_{S}}{\bigotimes_{S}} O_{S} \simeq R^{n} g_{*}\left(\Omega_{\dot{Y} / S}\right)
$$


$\bar{s}_{i}^{*}$ are the identity, and $\bar{s}_{i}^{*}$ vanish on $R^{a} g_{*}\left(\Omega_{Y / S}^{*}\right) \otimes_{o_{s}} R^{b} f_{*}^{\prime}\left(\Omega_{\mathscr{G} / S}^{*}\right)$ for $b>0$. Therefore $\bar{s}_{i}^{*}: R^{n} h_{*}\left(\Omega_{X / S}^{*}\right) \rightarrow R^{n} g_{*}\left(\Omega_{Y / S}^{*}\right)$ are nothing but the projection

$$
R^{n} h_{*}\left(\Omega_{X / S}^{*}\right) \longrightarrow R^{n} g_{*}\left(\Omega_{Y / S}^{\cdot}\right) \otimes R^{0} f_{*}^{\prime}\left(\Omega_{\mathscr{C} / S}^{*}\right) \simeq R^{n} g_{*}\left(\Omega_{Y / S}^{\cdot}\right),
$$

hence, $\bar{s}_{1}^{*}=\bar{s}_{2}^{*}$ on $E_{1}$-term.

In other cases, we shall show that $\bar{s}_{1}^{*}=\bar{s}_{2}^{*}$ on $E_{2}$-term. It suffices to see

$$
\bar{s}_{1}^{*}=\bar{s}_{2}^{*}: R^{n} h_{a *}\left(\mu_{X}\right) \longrightarrow R^{n} g_{a *}\left(\mu_{Y}\right) .
$$

By proper base change theorem, it is sufficient to verify that, for every (geometric) point $\bar{s}$ of $S$,

$$
\left(\bar{s}_{1}\right)_{s}^{*}=\left(\bar{s}_{2}\right)_{s}^{*}: H^{n}\left(X_{\bar{s}}, \mu\right) \longrightarrow H^{n}\left(Y_{\bar{s}}, \mu\right) .
$$

We have $X_{\bar{s}}=Y_{\bar{s}} \times \mathscr{C}_{\bar{s}}$. Since $H^{b}\left(\mathscr{C}_{\bar{s}}, A\right)$ are free $A$-modules, by Künneth formula, we get

$$
H^{n}\left(X_{\bar{s}}, \mu\right)=\bigoplus_{a+b=n} H^{a}\left(Y_{\bar{s}}, \mu\right) \underset{\boldsymbol{A}}{\otimes} H^{b}\left(\mathscr{C}_{\bar{s}}, A\right) .
$$

The rest of the proof is similar to that in the case a): On $H^{n}\left(Y_{\bar{s}}, \mu\right) \otimes$ $H^{0}\left(\mathscr{C}_{\bar{s}}, A\right)=H^{n}\left(Y_{\bar{s}}, \mu\right),\left(\bar{s}_{i}\right)_{\bar{s}}^{*}$ are the identity, and on $H^{a}\left(Y_{\bar{s}}, \mu\right) \otimes H^{b}\left(\mathscr{C}_{\bar{s}}, A\right)$, $\left(\bar{s}_{i}\right)_{s}^{*}$ vanish for $b>0$. Thus, $\bar{s}_{1}^{*}=\bar{s}_{2}^{*}$ on $E_{2}$-term.

Proposition (2.5). Let $S$ be a smooth scheme over $k, \mathscr{C}_{i} \rightarrow S(i=$ $1, \cdots, \ell)$ be the families of curves,

$$
X=\mathscr{C}_{1} \underset{S}{\times} \cdots \underset{S}{\times} \mathscr{C}_{\ell} .
$$

Let $s_{i}^{(0)}, s_{i}^{(1)}(i=1, \cdots, \ell)$ be sections of $\mathscr{C}_{i} / S . \quad$ For $\sigma \in 2^{[1, \ell]}=$ the set of maps from $[1, \ell]$ to $\{0,1\}$, we set

$$
\begin{aligned}
& |\sigma|=\sigma(1)+\cdots+\sigma(\ell) ; \\
& s^{\sigma}=s_{1}^{(\sigma(1))} \underset{S}{\times} \cdots \underset{S}{\times} s_{\ell}^{(\sigma(\ell))}: S \longrightarrow X,
\end{aligned}
$$

a section of $X / S$. Let $\operatorname{gr}^{p} H^{n}\left(X, \mu_{X}\right)=N^{p} H^{n}\left(X, \mu_{X}\right) / N^{p+1} H^{n}\left(X, \mu_{X}\right)$ be as in Section 1. For each $\sigma$, we get

$$
\left(s^{\sigma}\right)^{*}: \operatorname{gr}^{0} H^{n}\left(X, \mu_{X}\right) \longrightarrow \operatorname{gr}^{0} H^{n}\left(S, \mu_{S}\right) .
$$

Then, for $n<\ell$, we have

$$
0=\sum_{\sigma \in 2[1, \ell]}(-1)^{|\sigma|}\left(s^{\sigma}\right)^{*}: \operatorname{gr}^{0} H^{n}\left(X, \mu_{X}\right) \longrightarrow \operatorname{gr}^{0} H^{n}\left(S, \mu_{S}\right)
$$

Proof. Put $X_{0}=S, X_{i}=X_{i-1} \underset{S}{\times} \mathscr{C}_{i}(1 \leqslant i \leqslant \ell)$, so that $X_{\ell}=X$ and we have a diagram 


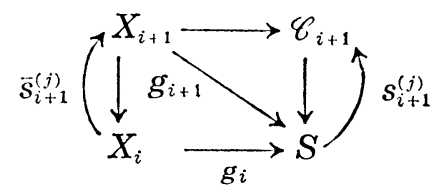

( $i=0, \cdots, \ell-1 ; j=0,1), \bar{s}_{i+1}^{(j)}$ being the base change of $s_{i+1}^{(j)}$.

First of all, we claim:

$$
0=\sum_{\sigma}(-1)^{|\sigma|}\left(s^{\sigma}\right)^{*}: R^{n}\left(\alpha_{S} \circ\left(g_{\ell}\right)_{a}\right)_{*}\left(\mu_{X}\right) \longrightarrow R^{n} \alpha_{S^{*}}\left(\mu_{S}\right)
$$

for $n<\ell$. We have the maps

$$
\left(\bar{s}_{i+1}^{(j)}\right)^{*}: R^{n}\left(\alpha_{S} \circ g_{i+1}\right)_{*}\left(\mu_{X_{i+1}}\right) \longrightarrow R^{n}\left(\alpha_{S} \circ g_{i}\right)_{*}\left(\mu_{X_{i}}\right)
$$

(we write $g_{i}$ for $\left(g_{i}\right)_{a}$, for the moment, to simplify the notation), hence,

$$
\Delta_{i+1}=\left(\bar{s}_{i+1}^{(1)}\right)^{*}-\left(\bar{s}_{i+1}^{(0)}\right)^{*}: R^{n}\left(\alpha_{S} \circ g_{i+1}\right)_{*}\left(\mu_{X_{i+1}}\right) \longrightarrow R^{n}\left(\alpha_{S} \circ g_{i}\right)_{*}\left(\mu_{X_{i}}\right) .
$$

Clearly, we have

$$
\Delta_{1} \circ \cdots \circ \Delta_{\ell}=\sum_{\sigma}(-1)^{|\sigma|}\left(s^{\sigma}\right)^{*}
$$

By the lemma (2.4),

$$
\Delta_{\imath+1}\left(g_{i+1} F^{p} R^{n}\left(\alpha_{S} \circ g_{i+1}\right)_{*}\left(\mu_{X_{i+1}}\right)\right) \subset{ }_{g_{i}} F^{p+1} R^{n}\left(\alpha_{S} \circ g_{i}\right)_{*}\left(\mu_{X_{i}}\right) .
$$

Hence,

$$
\Delta_{1} \circ \cdots \circ \Delta_{\ell}\left(R^{n}\left(\alpha_{S} \circ g_{\ell}\right)_{*}\left(\mu_{X}\right)\right) \subset{ }_{\mathrm{id}_{S}} F^{\ell} R^{n} \alpha_{S^{*}}\left(\mu_{S}\right) .
$$

Since the spectral sequences (2.2.1) and (2.3.1) are of first quadrant, we have ${ }_{\text {id }_{S}} F^{\ell} R^{n} \alpha_{S^{*}}\left(\mu_{S}\right)=0$ for $\ell>n$, whence the claim.

We set $g=g_{\ell}$. Since $\alpha_{S} \circ g_{a}=g \circ \alpha_{S}$, we have another Leray spectral sequence

$$
{ }_{g}^{N} E: E_{2}^{p, q}=R^{p} g_{*} R^{q} \alpha_{X *}\left(\mu_{X}\right) \Longrightarrow R^{n}\left(g \circ \alpha_{X}\right)_{*}\left(\mu_{X}\right)=R^{n}\left(\alpha_{S} \circ g_{a}\right)_{*}\left(\mu_{X}\right) .
$$

If $S=$ Spec $k$, this is no other than the spectral sequence

$$
E_{2}^{p, q}=H^{p}\left(X, R^{q} \alpha_{X}\left(\mu_{X}\right)\right) \Longrightarrow H^{n}\left(X_{a}, \mu_{X}\right),
$$

in Section 1. The spectral sequence ${ }_{g}^{N} E$ is obtained by sheafifying this spectral sequence with respect to "the Zariski topology of $S$ ".

For $\sigma \in 2^{[1, \ell]}$, we have again the commutative diagram 


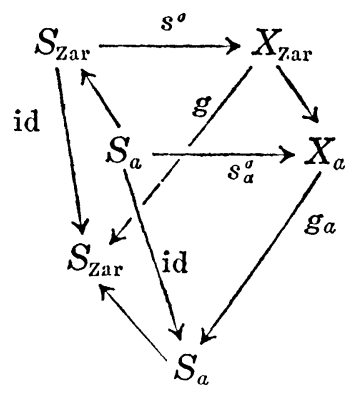

and a morphism $\left(s^{\sigma}\right)^{*}$ of spectral sequences from ${ }_{g}^{N} E$ to

$$
{ }_{\mathrm{id}, S}^{N} E: E_{2}^{p, q}=R^{p}\left(\mathrm{id}_{S}\right)_{*} R^{q} \alpha_{S *}\left(\mu_{S}\right) \Longrightarrow R^{n} \alpha_{S *}\left(\mu_{S}\right),
$$

which is degenerate: $E_{2}^{p, q}=0$ for $p \neq 0$. In particular, we have a commutative diagram

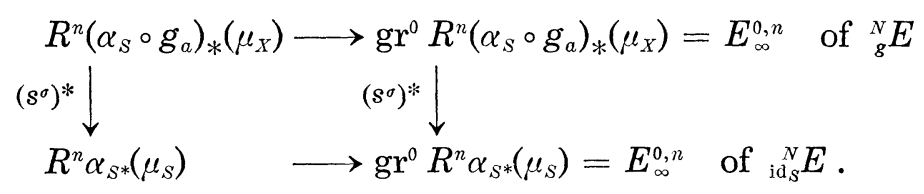

The horizontal map above is surjective since ${ }_{g}^{N} E$ is a first quadrant spectral sequence and the horizontal map below is an isomorphism by degeneracy of ${ }_{\mathrm{id} S}^{N} E$. From our claim above results that

$$
\Sigma=\sum_{\sigma}(-1)^{|\sigma|}\left(s^{\sigma}\right)^{*}: \operatorname{gr}^{0} R^{n}\left(\alpha_{S} \circ g_{a}\right)_{*}\left(\mu_{X}\right) \longrightarrow \operatorname{gr}^{0} R^{n} \alpha_{S^{*}}\left(\mu_{S}\right)
$$

is zero. Applying the functor $\Gamma(S, ?)$, we get

$$
0=\Sigma: \Gamma\left(S, \operatorname{gr}^{0} R^{n}\left(\alpha_{S} \circ g_{a}\right)_{*}\left(\mu_{X}\right)\right) \longrightarrow \Gamma\left(S, \operatorname{gr}^{0} R^{n} \alpha_{S^{*}}\left(\mu_{S}\right)\right) \text {. }
$$

As mentioned above, $\operatorname{gr}^{0} R^{n}\left(\alpha_{S} g_{a}\right)_{*}\left(\mu_{X}\right)$ is the sheafification (with respect to $S_{\text {Zar }}$ ) of the presheaf

$$
S^{\prime} \longmapsto \operatorname{gr}^{0} H^{n}\left(g^{-1}\left(S^{\prime}\right), \mu_{X}\right),
$$

hence we have a canonical map

$$
\operatorname{gr}^{0} H^{n}\left(X, \mu_{X}\right) \longrightarrow \Gamma\left(S, \operatorname{gr}^{0} R^{n}\left(\alpha_{S} \circ g_{a}\right)_{*}\left(\mu_{X}\right)\right),
$$

and the diagram

$$
\begin{aligned}
& \operatorname{gr}^{0} H^{n}\left(X, \mu_{X}\right) \longrightarrow \Gamma\left(S, \operatorname{gr}^{0} R^{n}\left(\alpha_{S} \circ g_{a}\right)_{*}\left(\mu_{X}\right)\right) \\
& {\left(s^{\sigma}\right)^{*}}^{\downarrow} \\
& \left.\operatorname{gr}^{0} H^{n}\left(S, \mu_{S}\right) \longrightarrow \Gamma\left(S, \operatorname{gr}^{0}\right)^{*} R^{n} \alpha_{S^{*}}\left(\mu_{S}\right)\right)
\end{aligned}
$$


commutes. We get therefore a commutative diagram

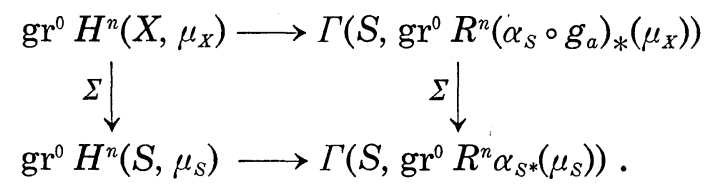

$R^{n} \alpha_{S^{*}}\left(\mu_{S}\right) \simeq \operatorname{gr}^{0} R^{n} \alpha_{S^{*}}\left(\mu_{S}\right)$ and the horizontal map below is nothing but

$$
E_{\infty}^{0, n} \longrightarrow E_{2}^{0, n}
$$

for the spectral sequence

$$
H^{p}\left(S, R^{q} \alpha_{S^{*}}\left(\mu_{S}\right)\right) \Longrightarrow H^{n}\left(S, \mu_{S}\right) ;
$$

so it is injective. Since the right vertical arrow is zero, so is the left vertical arrow.

$$
\text { q.e.d. }
$$

(2.6) In the case c), $\mu_{X}=\left(\mu_{\nu N}\right)^{\otimes a}$. Recall that the sheaves $\left(\left(\mu_{\nu N}\right)^{\otimes a}\right)_{N \in N}$ forms a projective system, so that we get a projective system $\left(H^{n}(X\right.$, $\left.\left.\left(\mu_{\nu N}\right)^{\otimes a}\right)\right)_{n \in N}$ and the $\nu$-adic etale cohomology is defined by

$$
H^{n}\left(X, Z_{\nu}(a)\right)=\varliminf^{n} H^{n}\left(X,\left(\mu_{\nu N}\right)^{\otimes a}\right) .
$$

Clearly, the subgroups $N^{p} H^{n}\left(X,\left(\mu_{\nu N}\right)^{\otimes a}\right)$ form a projective system, and we set:

$$
N^{p} H^{n}\left(X, Z_{\nu}(a)\right)=\varliminf_{\lim } N^{p} H^{n}\left(X,\left(\mu_{\nu N}\right)^{\otimes a}\right) .
$$

Then $N^{p} H^{n}\left(X, Z_{\nu}(a)\right)$ is a subgroup of $H^{n}\left(X, Z_{\nu}(a)\right)$, and they define a filtration on $H^{n}\left(X, Z_{\nu}(a)\right)$. We put:

$$
\operatorname{gr}^{p} H^{p}\left(X, Z_{\nu}(a)\right)=N^{p} H^{n}\left(X, Z_{\nu}(a)\right) / N^{p+1} H^{n}\left(X, Z_{\nu}(a)\right) .
$$

Since $H^{n}\left(X,\left(\mu_{\nu N}\right)^{\otimes a}\right)$ are finite groups, a projective subsystem of $\left(H^{n}(X\right.$, $\left.\left.\left(\mu_{\nu N}\right)^{\otimes a}\right)\right)_{N \in N}$ satisfies the Mittag-Leffler condition, so that

$$
\operatorname{gr}^{p} H^{n}\left(X, Z_{\nu}(a)\right)=\varliminf_{\lim } \operatorname{gr}^{p} H^{n}\left(X,\left(\mu_{\nu N}\right)^{\otimes a}\right) \text {. }
$$

For a cycle $Z$ on $X$ of codimension $p$, we have its fundamental class in $H^{2 p}\left(X, Z_{\nu}(p)\right)$ as the limit of those in $H^{2 p}\left(X,\left(\mu_{\nu N}\right)^{\otimes p}\right)$, which is compatible with direct images, pull-backs, and the intersections.

By taking the projective limit, we have an analogue of (1.5) for $N^{p} H^{n}\left(X, Z_{\nu}(a)\right)$. Also the analogues of (1.6), (1.7) and (2.5) hold. The cohomology theory $H^{n}\left(X, Z_{\nu}(a)\right)$ with this filtration will be referred to as the case $\left.c^{\prime}\right)$ in the sequel. 
Remark (2.6.1). On the other hand, we have another filtration ${ }^{\prime} N^{p} H^{n}\left(X, Z_{\nu}(a)\right)$ of $H^{n}\left(X, Z_{\nu}(a)\right)$ defined by

$$
{ }^{\prime} N^{p} H^{n}\left(X, Z_{\nu}(a)\right)=\sum_{X \in Z^{p}} \operatorname{Ker}\left(H^{n}\left(X, Z_{\nu}(a)\right) \longrightarrow H\left(X \backslash Y, Z_{\nu}(a)\right)\right)
$$

(cf. (1.1.7)), which should be called the coniveau filtration for $H^{n}\left(X, Z_{\nu}(a)\right)$. In general, we have

$$
{ }^{\prime} N^{p} H^{n}\left(X, Z_{\nu}(a)\right) \subset N^{p} H^{n}\left(X, Z_{\nu}(a)\right),
$$

and the equality holds for $n=2 p$ (both generated by the fundamental classes). The author does not know whether the equality holds in general or not.

\section{§3. Theorem}

(3.1) Let $T$ be a smooth quasi-projective variety. We denote the Chow group of $T$ of codimension $p$ by $C H^{p}(T)$, and put

$$
N^{p} H^{n}(T, a)=N^{p} H^{n}(T)=N^{p}(T, \mu(a)) \otimes \boldsymbol{Q},
$$

where $N^{p} H^{N}(T, \mu(a))$ is one of the cohomology theories a), b) and $c^{\prime}$ ) (cf. (2.6)). Let $V$ be a smooth projective variety. If $z \in C H^{p}(T \times V)$, we denote by $\{z\}$ its fundamental class in $N^{p} H^{2 p}(T \times V, p) \simeq \operatorname{gr}^{p} H^{2 p}(T \times V, p)$. Set $r=\operatorname{dim} V-p$; then we have the map

$$
\left\{{ }^{t} z\right\}: \operatorname{gr}^{r} H^{2 r+\ell}(V, a) \longrightarrow \operatorname{gr}^{0} H^{\ell}(T, a-r)
$$

defined by $\left\{{ }^{t} z\right\}(\omega)=\operatorname{pr}_{T *}\left(\{z\} \cup \operatorname{pr}_{V}^{*}(\omega)\right)$, where $\operatorname{pr}_{T}$ and $\operatorname{pr}_{V}$ are the projections from $T \times V$.

For $t \in T$ (closed point), we denote by $i_{t}$ the injection $V \simeq t \times V \subset$ $T \times V$. Then we obtain the map $i_{t}^{*}: C H^{p}(T \times V) \rightarrow C H^{p}(V), z \mapsto i_{t}^{*}(z)=$ $z(t)$. Moreover, for an integer $\ell \geqslant 0$, let $F^{\ell} C H^{p}(V)$ denote the subgroup of $C H^{p}(V)$ consisting of cycles $\ell$-cube equivalent to zero modulo rational equivalence (cf. [9]).

THEOREM (3.2). Let $T$ be a smooth quasi-projective variety, $V$ a smooth projective variety of dimension $m, p$, $\ell$ integers $\geqslant 0, z \in C H^{p}(T \times V)$, and $r=m-p$. Suppose that the ground field $k$ is uncountable.

If $z(t) \in F^{\ell} C H^{p}(V)$ for all $t \in T$, then the map

$$
\left\{{ }^{t} z\right\}: \operatorname{gr}^{r} H^{2 r+\ell^{\prime}}(V, a) \longrightarrow \operatorname{gr}^{0} H^{\ell^{\prime}}(T, a-r)
$$

vanishes for $\ell^{\prime}<\ell$. 
(3.3). Before giving the proof, we shall recall about the Chow scheme, especially in positive characteristic (cf. [9], §1). Fix an embedding $V \subset \boldsymbol{P}^{N}$, and let $C_{r}(V)_{d}(d>0)$ denote the Chow scheme of effective $r$-cycles on $V$ of degree $d$ with respect to this embedding. If $Z$ is an effective cycle on $T \times V$ of codimension $p$ which is non-degenerate on $T$, and if the $r$-cycle $Z(t)$ on $V$ is of degree $d$ for general $t \in T$, then we get a rational map $f: T \cdots C_{r}(V)_{d}, t \mapsto Z(t)$. The map $Z \mapsto f$ is injective in arbitary characteristic, and bijective if char $k=0$. Moreover, if $\phi: T^{\prime} \rightarrow T$ is a morphism, the following are equivalent: (i) $(\phi \times \mathrm{id})^{*}(Z)$ is defined; (ii) $\operatorname{Im} \phi$ and the domain of definition of $f$ intersect. In this case, the rational map $f \circ \phi: T^{\prime} \ldots \rightarrow C_{r}(V)_{d}$ corresponds to the cycle $(\phi \times \mathrm{id})^{*}(Z)$. Hence if char $k=0$, we have the map

$$
\text { r: } \operatorname{Hom}_{\mathrm{rat}}\left(T, C_{r}(V)_{d}\right) \longrightarrow N^{p} H^{2 p}(T \times V)
$$

defined by $f \mapsto\{Z\}$, which is "functorial in $T$ ".

Suppose that char $k>0$, and we shall prove that, in this case, we have a map similar to $\gamma$ too. Recall that for a rational map $f: T \cdots \rightarrow$ $C_{r}(V)_{d}$, there exists a purely inseparable finite extension $L$ of $k(T)$ such that if $\psi: S \rightarrow T$ is a dominant morphism with $L \subset k(S)$, then there exists a cycle on $S \times V$ of codimension $p$ whose corresponding rational map $S \ldots \rightarrow C_{r}(V)_{d}$ is $f \circ \psi$. Let $q$ be a power of the characteristic of $k$, and $F$ : Spec $k \rightarrow \operatorname{Spec} k$ be the Frobenius morphism, i.e., $q$-th power map on the ring level. Put

$$
T^{(q)}=T \underset{\text { Spec } k^{F}}{\times} \operatorname{Spec} k
$$

and we get the relative Frobenius morphism $F_{q}: T \rightarrow T^{(q)}$. Then $k\left(T^{(q)}\right)$ $=k(T)^{q} \cdot k=k(T)^{q}$. Since $k$ is algebraically closed, $F$ is an isomorphism; we set

$$
T^{(1 / q)}=T \underset{\text { Spec } k^{F-1}}{\times} \operatorname{Spec} k
$$

Then $T \mapsto T^{(1 / q)}$ is functorial and $T^{(1 / q)}$ is smooth quasi-projective if so is T. From $\left(T^{(1 / q)}\right)^{(q)} \simeq T$, we get a morphism $F_{1 / q}: T^{(1 / q)} \rightarrow T$ and $k\left(T^{(1 / q}\right)=$ $k(T)^{1 / q}$. Thus for a rational map $f: T \cdots C_{r}(V)_{d}$, if $q$ is large enough, $L \subset k\left(T^{(1 / q)}\right)$ and there is a cycle $Z^{\prime}$ on $T^{(1 / q)} \times V$ of codimension $p$ whose corresponding rational map is $f \circ F_{1 / q}$, and such $Z^{\prime}$ is unique for each $q$. Then we set 


$$
\gamma(f)=\frac{1}{\operatorname{deg} F_{1 / q}}\left(F_{1 / q} \times \operatorname{id}_{V}\right)_{*}\left(\left\{Z^{\prime}\right\}\right) \in N^{p} H^{2 p}(T \times V) .
$$

The projection formula shows that $\gamma(f)$ is independent of choice of $q$, and we get a map

$$
\text { r: } \operatorname{Hom}_{\mathrm{rat}}\left(T, C_{r}(V)_{d}\right) \longrightarrow N^{p} H^{2 p}(T \times V),
$$

which is "functorial in $T$ " (Note that $F_{1 / q}^{*}: H^{n}(T \times V) \rightarrow H^{n}\left(T^{(1 / q)} \times V\right.$ ) is bijective and $\left.F_{1 / q}^{*} \circ F_{1 / q *}=\operatorname{deg} F_{1 / q}\right)$.

For char $k \geqslant 0$, if $f: T \cdots \rightarrow C_{r}(V)_{d}$ is a rational map, we obtain a map

$$
f^{\#}: \operatorname{gr}^{r} H^{2 r+\varepsilon^{\prime}}(V) \longrightarrow \operatorname{gr}^{0} H^{\ell^{\prime}}(T)
$$

by $f^{\sharp}(\omega)=\left\{{ }^{t} \gamma(f)\right\}(\omega)$. Evidently, we have the following formulae: if $g$ : $T \cdots C_{r}(V)_{d^{\prime}}$ is another rational map, we have

$$
(f+g)^{\#}=f^{\#}+g^{\#}: \operatorname{gr}^{r} H^{2 r+\ell^{\prime}}(V) \longrightarrow \operatorname{gr}^{0} H^{\ell^{\prime}}(T),
$$

where $f+g: T \cdots \rightarrow C_{r}(V)_{d+d^{\prime}}$ denotes the summation on the Chow schemes. If $\phi: T^{\prime} \rightarrow T$ is a morphism such that $f \circ \phi: T \cdots \rightarrow C_{r}(V)_{d}$ is defined,

$$
(f \circ \phi)^{\#}=\phi^{*} \circ f^{\sharp}: \operatorname{gr}^{r} H^{2 r+\ell^{\prime}}(V) \longrightarrow \operatorname{gr}^{0} H^{\ell^{\prime}}(T) .
$$

If $f: T \cdots \rightarrow C_{r}(V)_{d}$ corresponds to a cycle $Z$, then $f^{\sharp}=\left\{{ }^{t} Z\right\}$.

(3.4) We are now ready to prove the theorem (3.2). Let $Z$ be a representative of $z$. We may assume that every component of $Z$ is nondegenerate on $T$, for degenerate components have no effects on $z(t)$ and $\left\{{ }^{t} z\right\}$. Since the map induced by an open immersion on $\operatorname{gr}^{0} H^{\ell^{\prime}}$ is injective by (1.6), we can suppose that $i_{t}^{*}(Z)$ are defined for all $t \in T$. Let $Z^{+}$and $Z^{-}$be the positive and negative parts of $Z$ respectively. Clearly we can suppose $\ell>0$, and in that case, the degrees of $Z^{+}(t)$ and $Z^{-}(t)(t \in T)$ are equal, and we have the corresponding rational maps $f, g: T \rightarrow C_{r}(V)_{d}$, which are, in this case, morphisms. By hypothesis, the cycles $f(t)$ and $g(t)$ are $\ell$-cube equivalent for all $t \in T$. Recall the following theorem:

TheOREm (3.4.1. $=[9],(5.6)) . \quad$ Let $T$ be a smooth quasi-projective variety, $V$ a smooth projective variety, $f$ and $g$ are morphisms from $T$ to $C_{r}(V)_{d}$, and suppose that the ground field is uncountable. If $f(t)$ and $g(t)$ are $\ell$-cube equivalent, then there exist a smooth quasi-projective variety $S, a$ dominant morphism $e: S \rightarrow T$, families $\mathscr{C}_{i}$ of curves over $S$, sections $s_{i}^{(0)}$ and $s_{i}^{(1)}$ of $\mathscr{C}_{i} / S(1 \leqslant i \leqslant \ell)$ and a rational map $H^{\#}$ from $\mathscr{C}_{1} \underset{S}{\times} \cdots \underset{S}{\times} \mathscr{C}_{\ell}$ to 
$C_{r}(V)_{d^{\prime}} \times C_{r}(V)_{d^{\prime \prime}}$ such that

(i) for every $\sigma \in 2^{[1, \ell]}, s^{\sigma}:=s_{1}^{(\sigma(\ell))} \times \cdots \times s_{\ell}^{(\sigma(\ell))}$ has its image in the domain of definition of $H$;

(ii) as morphisms from $S$ to $C_{r}(V)_{N}\left(N=2^{\ell-1}\left(d^{\prime}+d^{\prime \prime}\right)+d\right)$,

$$
\begin{aligned}
f \circ e & +\sum_{|\sigma| \equiv 0} \mathrm{pr}_{1} \circ H \circ s^{\sigma}+\sum_{|\sigma| \equiv 1} \mathrm{pr}_{2} \circ H \circ s^{\sigma} \\
& =g \circ e+\sum_{|\sigma| \equiv 1} \mathrm{pr}_{1} \circ H \circ s^{\sigma}+\sum_{|\sigma| \equiv 0} \mathrm{pr}_{2} \circ H \circ s^{\sigma},
\end{aligned}
$$

where $|\sigma|=\sigma(1)+\cdots+\sigma(\ell)$, and " $\equiv "$ means "equivalent molulo 2".

As noted in [9], (1.3.1), the theorem is valid even in the positive characteristic case.

(3.4.2) We shall return to the proof of (3.2). Note that, as maps from $\operatorname{gr}^{r} H^{2 r+\ell^{\prime}}(V)$ to $\operatorname{gr}^{0} H^{\ell^{\prime}}(T),\left\{{ }^{t} z\right\}=\left\{{ }^{t} Z^{+}\right\}-\left\{{ }^{t} Z^{-}\right\}$and $f^{\#}=\left\{{ }^{t} Z^{+}\right\}, g^{\#}=$ $\left\{{ }^{t} Z^{-}\right\}$. By virtue of (1.7), it is therefore sufficient to show that $e^{*} \circ f^{\sharp}=$ $e^{*} \circ g^{\sharp}$, i.e. $(f \circ e)^{\sharp}=(g \circ e)^{\sharp}$. But by (ii) above, we have the equality

$$
\begin{aligned}
(f \circ e)^{\#} & +\sum_{|\sigma| \equiv 0}\left(\mathrm{pr}_{1} \circ H \circ s^{\sigma}\right)^{\#}+\sum_{|\sigma| \equiv 1}\left(\mathrm{pr}_{2} \circ H \circ s^{\sigma}\right)^{\#} \\
= & (g \circ e)^{\#}+\sum_{|\sigma| \equiv 1}\left(\mathrm{pr}_{1} \circ H \circ S^{\sigma}\right)^{\#}+\sum_{|\sigma| \equiv 0}\left(\mathrm{pr}_{2} \circ H \circ s^{\sigma}\right)^{\sharp},
\end{aligned}
$$

or, by (i),

$$
(f \circ e)^{\#}+\sum_{\sigma}(-1)^{|\sigma|} s^{\sigma_{*}} \circ\left(\mathrm{pr}_{1} \circ H\right)^{\#}=(g \circ e)^{\#}+\sum_{\sigma}(-1)^{|\sigma|} s^{\sigma_{*}} \circ\left(\mathrm{pr}_{2} \circ H\right)^{\#} .
$$

Since we have $\sum_{\sigma}(-1)^{|\sigma|} s^{\sigma_{*}}=0$ by $(2.5)$, we obtain $(f \circ e)^{\#}=(g \circ e)^{\#}$. q.e.d.

In the sequel, $k$ is supposed to be uncountable (except (3.6.1)). Recall that $\operatorname{gr}^{\ell} C H^{p}(V)=F^{\ell} C H^{p}(V) / F^{\ell+1} C H^{p}(V)$.

CoRollary (3.5). Let $T$ be an abelian variety or a product of (smooth complete) curves, $V$ a smooth projective variety, $z \in \mathrm{gr}^{0} \mathrm{CH}^{p}(T \times V), r+p=$ $\operatorname{dim} V, \ell$ an integer. If

$$
[z]: \operatorname{gr}^{\ell} C H_{0}(T) \longrightarrow \operatorname{gr}^{\ell} C H^{p}(V), \quad \gamma \longmapsto z(\gamma)
$$

is zero, then

$$
\left\{{ }^{t} z\right\}: \operatorname{gr}^{r} H^{2 r+\ell}(V, a) \longrightarrow \operatorname{gr}^{0} H^{\ell}(T, a-r)
$$

vanishes.

The general idea of our proof is as follows: Let $x \in C H^{t}(S \times T), t=$ $\operatorname{dim} T$. If $x(s) \in F^{\ell} C H_{0}(T)$ for all $s \in S$, and if 


$$
0=\left\{{ }^{t} x\right\} \circ\left\{{ }^{t} z\right\}: \operatorname{gr}^{r} H^{2 r+\ell}(V) \longrightarrow \operatorname{gr}^{0} H^{\ell}(T)
$$

implies $\left\{{ }^{t} z\right\}=0$, then we get the corollary, since we have $\left\{{ }^{t} x\right\} \circ\left\{{ }^{t} z\right\}=0$ by the theorem (3.2).

(i) Suppose that $T$ is an abelian variety. For an integer $n$, let $n_{T}$ : $T \rightarrow T$ denote the multiplication by $n, \Gamma_{n}$ its graph. Then for $t \in T, \Gamma_{n}(t)$ $=n_{T *}(t)=(n t) \in C H_{0}(T)$ and

$$
\left\{{ }^{t} \Gamma_{n}\right\}: H^{\ell}(T) \longrightarrow H^{\ell}(T)
$$

is multiplication by $n^{\ell}$. Let

$$
X=\sum_{n=0}^{\ell}(-1)^{\ell-n}\left(\begin{array}{l}
\ell \\
n
\end{array}\right) \Gamma_{n} .
$$

For $t \in T$,

$$
X(t)=\sum_{n=0}^{\ell}(-1)^{\ell-n}\left(\begin{array}{l}
\ell \\
n
\end{array}\right) \Gamma_{n}(t)=((t)-(0)) * \cdots *((t)-(0)) \quad(\ell \text { times })
$$

by "binomial theorem", where $*$ denotes the Pontrjagin product. Hence $\operatorname{Im}[X] \subset F^{\ell} C H_{0}(T)$, and $\left\{{ }^{t} X\right\}: H^{\ell}(T) \rightarrow H^{\ell}(T)$ is multiplication by

$$
\sum_{n=0}^{\ell}(-1)^{\ell-n}\left(\begin{array}{l}
\ell \\
n
\end{array}\right) n^{\ell}=\ell !
$$

hence $\left\{{ }^{t} X\right\}$ is bijective on $\operatorname{gr}^{0} H^{\ell}(T)$.

(ii) Suppose that $T$ is a product of curves. First, assume $T=$ $C_{1} \times \cdots \times C_{\ell}$, a product of $\ell$ curves. For each $i$, take a point $a_{i} \in C_{i}$. Consider the algebraic correspondence $X$ defined by

$$
T \ni t=\left(t_{1}, \cdots, t_{\ell}\right) \longrightarrow\left(\left(t_{1}\right)-\left(a_{1}\right)\right) \times \cdots \times\left(\left(t_{\ell}\right)-\left(a_{\ell}\right)\right) .
$$

Clearly $X(t) \in F^{\ell} C H_{0}(T)$ for $t \in T$, and

$$
\left\{{ }^{t} X\right\}: H^{\ell}(T) \longrightarrow H^{\ell}(T)
$$

is the identity, hence so is $\left\{{ }^{t} X\right\}$ on $\operatorname{gr}^{0} H^{\ell}(T)$. The case of a product of $\ell$ curves is proven. In general, if $\operatorname{dim} T<\ell, \operatorname{gr}^{0} H^{\ell}(T)=0$. Thus we may assume:

$$
T=C_{1} \times \cdots \times C_{n} \quad\left(n>\ell, C_{i} \text { : curves }\right) .
$$

For $I \subset[1, n]$, card $I=\ell$, let $\mathrm{pr}_{I}: T \rightarrow C_{I}=\prod_{i \in I} C_{i}$ denote the projection. It induces a map $\operatorname{pr}_{I}^{*}: \operatorname{gr}^{0} H^{\ell}\left(C_{I}\right) \rightarrow \operatorname{gr}^{0} H^{\ell}(T)$ and we have an isomorphism

$$
\underset{\operatorname{card} I=\ell}{\oplus} \operatorname{gr}^{0} H^{\ell}\left(C_{I}\right) \stackrel{\sim}{\longrightarrow} \operatorname{gr}^{0} H^{\ell}(T) .
$$


The inverse is given as follows: choose a point $a_{i} \in C_{i}$ on each curve, and let $j_{I}: C_{I} \rightarrow T$ be the embedding obtained by "inserting $a_{i}$ " for $i \notin I$. The map $j_{I}^{*}: \operatorname{gr}^{0} H^{\ell}(T) \rightarrow \operatorname{gr}^{0} H^{\ell}\left(C_{I}\right)$ is the $I$-component of the inverse of the isomorphism above. In fact, by Künneth formula,

$$
\underset{a_{1}+\cdots+a_{n}=\ell}{\oplus} H^{a_{1}}\left(C_{1}\right) \otimes \cdots \otimes H^{a_{n}}\left(C_{n}\right) \stackrel{\sim}{\longrightarrow} H^{\ell}(T) .
$$

The map is induced by the projections (and the cup-product). Note that $H^{2}\left(C_{i}\right)=N^{1} H^{2}\left(C_{i}\right)$. Hence if $a_{1}+\cdots+a_{n}=\ell$ and $a_{i}=2$ for some $i$, the image of $H^{a_{1}}\left(C_{1}\right) \otimes \cdots \otimes H_{a_{n}}\left(C_{n}\right)$ in $H^{\ell}(T)$ lies in $N^{1} H^{\ell}(T)$, and

$$
\oplus H^{a_{1}}\left(C_{1}\right) \otimes \cdots \otimes H^{a_{n}}\left(C_{n}\right) \longrightarrow \operatorname{gr}^{0} H^{\ell}(T)
$$

is surjective, where the summation is over such $\left(a_{i}\right)$ that $a_{1}+\cdots+a_{n}$ $=\ell$ and $a_{i}=0$, or 1 . For such $\left(a_{i}\right)$, put $I=\left\{i ; a_{i}=1\right\}$, and we have the isomorphism

$$
H^{e}\left(C_{I}\right) \stackrel{\mathrm{pr}_{I}}{\longrightarrow} H^{e}(T) \stackrel{\text { proj. }}{\longrightarrow} H^{a_{1}}\left(C_{1}\right) \otimes \cdots \otimes H^{a_{n}}\left(C_{n}\right) .
$$

Hence, the map (3.5.1) is surjective. The map

$$
H^{\ell}\left(C_{I}\right) \stackrel{\operatorname{pr}_{I}^{*}}{\longrightarrow} H^{\ell}(T) \stackrel{j_{I^{\prime}}^{*}}{\longrightarrow} H^{\ell}\left(C_{I^{\prime}}\right)
$$

vanishes if $I \neq I^{\prime}$, and is an identity if $I=I^{\prime}$. This shows that (3.5.1) is an isomorphism.

Returning to the proof of the corollary (3.5), suppose that

$$
\left\{{ }^{t} z\right\}: \operatorname{gr}^{r} H^{2 r+\ell}(V) \longrightarrow \operatorname{gr}^{0} H^{\ell}(T)
$$

is non-zero. Then for some $I$, with $\operatorname{card} I=l$,

$$
j_{I}^{*} \circ\left\{{ }^{t} z\right\}: \operatorname{gr}^{r} H^{2 r+\ell}(V) \longrightarrow \operatorname{gr} H^{\ell}\left(C_{I}\right)
$$

is also non-zero. Let $\gamma$ be the graph of $j_{I}$ in $C H^{n}\left(C_{I} \times T\right)$ and set $x=$ $z \circ \gamma \in C H^{p}\left(C_{I} \times V\right)$. Then

$$
[x]=[z] \circ j_{I^{*}}: \operatorname{gr}^{\ell} C H_{0}\left(C_{I}\right) \longrightarrow \operatorname{gr}^{\ell} C H^{p}(V)
$$

is zero, but

$$
\left\{{ }^{t} x\right\}=j_{I}^{*} \circ\left\{{ }^{t} z\right\}: \operatorname{gr}^{r} H^{2 r+\ell}(V) \longrightarrow \operatorname{gr}^{0} H^{\ell}\left(C_{I}\right)
$$

is not zero, which is absurd.

Remark (3.5.2). The author does not know whether or not the corollary remains true without the assumption on $T$. The argument similar 
to the one just above shows, however, that the corollary holds for a smooth projective variety $T$ satisfying the following condition: for any non-zero $x \in \operatorname{gr}^{0} H^{e}(T)$, there exist a product of curves (or an abelian variety) $T^{\prime}$ and $y \in C H^{t}\left(T \times T^{\prime}\right)(t=\operatorname{dim} T)$ such that

$$
\{y\}(x) \neq 0 \quad \text { in } \operatorname{gr}^{0} H^{\ell}\left(T^{\prime}\right) .
$$

For example, a variety dominated by a product of curves or an abelian variety is such a variety.

Corollary (3.6). If $V$ is an abelian variety or a product of curves, then $\operatorname{gr}^{0} H^{\ell}(V) \neq 0$ implies $\operatorname{gr}^{\ell} C H_{0}(V) \neq 0$. In particular, there exists an abelian variety in any characteristic such that $\mathrm{F}^{2} \mathrm{CH}_{0}(V) \neq F^{3} \mathrm{CH}_{0}(V)$.

Apply the corollary (3.5) to $T=V, z=$ the diagonal of $V \times V$; then $z={ }^{t} z$ induces the identity on $\operatorname{gr}^{\ell} \mathrm{CH}_{0}(V)$ and on $\operatorname{gr}^{0} H^{\ell}(V)$. Notice that $\operatorname{dim} \operatorname{gr}^{0} H^{2}(V)=\left(2^{\text {nd }}\right.$ Betti number $)$-(Picard number). If char $k=0$, then, $\operatorname{gr}^{0} H^{2}(V) \neq 0$ (even $\operatorname{gr}^{0} H^{n}(V, \boldsymbol{Q}) \neq 0$ for $n \leqslant \operatorname{dim} V$ ) for any abelian variety. In positive characteristic, $\operatorname{gr}^{0} H^{2}(V) \neq 0$ if an abelian variety $V$ is not supersingular.

Remark (3.6.1). For a supersingular abelian variety $A$, we have $F^{1} C H_{0}(A) \simeq A$ (N. Maruyama [8]), and $\operatorname{gr}^{2} C H_{0}(A)=0$. Thus, contrary to the case of characteristic 0 , we cannot conclude that $H^{0}\left(A, \Omega^{2}\right) \neq 0$ implies $\operatorname{gr}^{2} C H_{0}(A) \neq 0$. Also notice that if $k=\bar{F}_{q}$, the algebraic closure of a finite field, we have $F^{2} C H^{p}(V)=0$ for any smooth projective variety $V$ and any $p$. The assumption that $k$ is uncountable is thus not unnecessary (if a weaker condition can be good).

The author does not know whether or not there exists an abelian variety (in positive characteristic) for which $\operatorname{gr}^{0} H^{\ell}\left(A, \boldsymbol{Q}_{\nu}\right) \neq 0$ for $l \leqslant \operatorname{dim} A$, though it is plausible for general abelian variety.

Remark (3.7). As mentioned in the introduction, we proved an analogue of (3.2) in [9] for the Hodge cohomology, whose proof was not purely algebraic. We can give an algebraic proof by a similar method as in Section 2.

\section{References}

[1 ] Atiyah, M. F., and Hodge, W. V. D., Integrals of the second kind on an algebraic variety, Ann. of Math., 62 (1955), 56-91.

[2] Bloch, S., Lectures on algebraic cycles, Duke University Mathematics Series, IV, 1980 . 
[ 3 ] Bloch, S., and Ogus, A., Gersten's conjecture and the homology of schemes, Ann. Sci. École Norm. Sup., 7 (1974), 181-201.

[ 4 ] Cartan, H., and Eilenberg, S., Homological Algebra, Princeton University Press, Princeton, 1956.

[5] Grothendieck, A., Le Group de Brauer III, in Dix exposes sur la cohomologie des schemas, North Holland, Amsterdam, 1968.

[6] Hartshorne, R., Residues and Duality, Lecture Notes in Mathematics, 20, Springer Verlag, Berlin-Heidelberg-New York, 1966.

[ 7 ] Katz, N., Nilpotent connections and the monodromy theorem; an application of a result of Turrittin, Publ. Math. I.H.E.S., 39 (1970), 175-232.

[8] Maruyama, N., On 0-cycles on abelian varieties (in Japanese), 24th Daisugaku symposium hokokushu, Algebraic Geometry and Ring Theory, July, 25-27, 1978, Yamaguchi-ken shakai-hukushi kaikan, 29-36.

[ 9 ] Saito, H., The Hodge cohomology and cubic equivalences, Nagoya Math. J., 94 (1984), 1-41.

Department of Mathematics

Faculty of Science

Nagoya University

Chikusa-ku, Nagoya 464

Japan 